Revista Internacional de Sociología (RIS)

Vol.67, N² 2, MaYo-Agosto, 437-456, 2009

ISSN: $0034-9712$

DOI: $10.3989 /$ ris.2008.11.24

\title{
POR UN GIRO ANALÍTICO EN SOCIOLOGÍA
}

\section{ARGUING FOR AN ANALYTICAL TURN IN SOCIOLOGY}

\author{
FERnANDo Aguiar \\ Instituto de Estudios Sociales Avanzados (IESA-CSIC), Córdoba, España \\ faguiar@iesa.csic.es
}

\author{
ANDRÉS dE FRANCISCO \\ Universidad Complutense de Madrid, España \\ adefrancisco@cps.ucm.es \\ José Antonio Noguera \\ Universidad Autónoma de Barcelona, España \\ jose.noguera@uab.cat
}

\section{RESUMEN}

En los últimos años un importante grupo de científicos sociales ha sentido la necesidad de promover un giro analítico en sociología. Esa necesidad se ha debido al hecho de que algunas corrientes sociológicas muy influyentes se han deslizado por la pendiente del irracionalismo, la imprecisión conceptual, la vaguedad teórica y la esterilidad científica y explicativa. El giro analítico supondría, pues, sentar la sociología de nuevo sobre una base sólida. La sociología española no ha sido ajena ni al deterioro de la sociología como disciplina científica ni al giro analítico. Por tal motivo el presente artículo quiere contribuir al debate actual mediante la disección, primero, del estado actual de la sociología y estableciendo después las bases de lo que es la sociología analítica, para finalizar defendiéndola frente a algunas confusiones y acusaciones habituales.

\section{Palabras Clave Adicionales}

Microfundamentos, Pluralismo metodológico, Racionalidad, Sociología analítica, Sociología normativa.

\section{Abstract}

In the last years an important group of social scientists has felt the need to promote an analytical turn in sociology. This need is due to the fact that some influential sociological trends have slid down the slope of irrationalism, conceptual imprecision, theoretical vagueness and scientific and explanatory futility. The analytical turn would imply, then, to set sociology on a sound basis again. Spanish sociology has been alien neither to the declining of sociology as scientific discipline nor to the analytical turn. So this paper wants to contribute to the current debate starting from the dissection of the present state of the discipline, laying the foundations of analytical sociology, and, finally, defending it against frequent misreadings and accusations.

\section{Additional KeYWORdS}

Analytical Sociology, Methodolgoical pluralism, Microfoundations, Normative sociology, Rationality. 


\section{INTRODUCCIÓN ${ }^{1}$}

La sociología analítica es ya un movimiento intelectual que despierta una creciente atención internacional. Las revistas más prestigiosas abren más y más sus páginas a artículos escritos dentro de esta línea, y el movimiento aumenta paulatinamente su visibilidad en congresos y reuniones científicas internacionales. La sociología analítica es una tendencia cada día más reconocida como intelectualmente fructífera y vigorosa, está contagiando ese vigor intelectual a muchos científicos sociales, y, en algunos casos, incluso institucionalizándolo en comunidades intelectuales amplias. Organizaciones como la European Academy of Sociology y Departamentos de Sociología como los de las Universidades de Oxford, Estocolmo, Cornell o el GEMAS de la Sorbona agrupan a algunos de sus representantes más destacados, y está en marcha la creación de una red europea de sociología analítica. Diversos simposios y workshops se han llevado a cabo recientemente para impulsarla, con la participación de científicos sociales analíticos como Peter Hedström, Raymond Boudon, Jon Elster, Diego Gambetta, y otros muchos (la 1st Conference on Analytical Sociology and Social Mechanisms se celebró en Oxford en mayo de 2008; la segunda tendrá lugar en Turín en junio de 2009, y la tercera en Barcelona en 2010; también en octubre de 2008 el GEMAS de la Sorbona organizó en París un workshop internacional sobre Sociología Analítica). En España ya se ha creado un Grupo de Trabajo de Sociología Analítica dentro de la Federación Española de Sociología (FES), varios grupos de investigación trabajan en esta línea (como el GSADI, Grupo de Sociología Analítica y Diseño Institucional, de la Universidad Autónoma de Barcelona) ${ }^{2}$, se ha publicado un monográfico sobre sociología analítica (Papers, $\left.n^{\circ} 80,2006\right)$ y un monográfico sobre acción colectiva desde este enfoque (Revista Internacional de Sociología, n 46, 2007). En mayo de 2007 se celebró en Barcelona la I Jornada sobre Sociología Analítica y Diseño Institucional, y se prevé una segunda antes de finalizar el año 2009. ${ }^{3}$

El presente texto elabora los principios e intenciones del mencionado Grupo de Sociología Analítica de la FES.

\footnotetext{
${ }^{1}$ José Antonio Noguera agradece el apoyo del Ministerio de Educación y Ciencia a través del proyecto de I+D+i con referencia SEJ2006-00959/SOCl. Fernando Aguiar y Andrés de Francisco han contado también con ayuda pública para realizar este trabajo gracias al proyecto SEJ2006-07695. Fernando Aguiar agradece a los organizadores del IV Congreso Andaluz de Sociología (celebrado en Carmona, Sevilla, entre el 20 y el 22 de noviembre de 2008) la invitación para presentar una versión previa de este texto como conferencia inaugural.

${ }^{2}$ http://gsadi.uab.es

${ }^{3}$ Textos recientes de referencia para la sociología analítica son Hedström (2005, 2009), Barbera (2004) o Elster (2007).
} 


\section{UN DIAGNÓSTICO CRÍTICO DE LA SOCIOLOGÍA ACTUAL}

La ciencia moderna, aun con todas sus limitaciones y episodios oscuros, es el mayor proyecto de emancipación intelectual que la historia ha conocido. La sociología nació con vocación de disciplina científica y, por ello, con la intención de aplicar la racionalidad crítica y democrática de la ciencia al estudio de la realidad social. Sin embargo, dos siglos después de su nacimiento, la sociología no goza de una salud científica tan buena como sería deseable para una disciplina sin la cual no se puede comprender el mundo moderno. Lejos de ocupar el lugar que le corresponde como ciencia de la sociedad por excelencia, nos hemos acostumbrado a una sociología fragmentada, atrapada entre la timidez empírica y la grandilocuencia teórica, incapaz de conseguir acuerdos estables sobre sus fundamentos teóricos, epistémicos y metodológicos. Ello ha obstaculizado el surgimiento de una verdadera comunidad científica, entendida como comunidad de juicio crítico que comparte un vocabulario, unas prácticas y unos valores (sin que tal cosa excluya, claro está, discrepancias teóricas y empíricas).

Muy al contrario, algunos de los sociólogos más célebres, y muchos de sus seguidores por activa o por pasiva, parecen instalados hoy en un cómodo "todo vale" que no hace ascos a los géneros más diversos, desde el ensayismo social con vuelo literario o periodístico hasta la simple palabrería impresionista. Entre los sociólogos cuya influencia trasciende el ámbito académico cunde cada vez más, por desgracia, un diletantismo que no beneficia a la sociología como disciplina científica. La ocurrencia, el chispazo momentáneo, la metáfora aparentemente brillante que no contribuye al análisis riguroso de los problemas del mundo actual en cuanto se apaga su brillo, o la producción alegre de etiquetas lingüísticas o conceptuales se han convertido, en demasiados casos, en el rasgo más señalado y públicamente visible del quehacer sociológico ${ }^{4}$. Al respecto, ya dijo Max Weber,

"Casi todas las ciencias deben algo a los diletantes, muchas veces valiosos puntos de vista. Pero el diletantismo como principio de la ciencia sería su fin. Quien quiera ver "panoramas" que vaya al cine" (Weber, 1983: 21).

Que la sociología se haya convertido en una empresa "a la carta", tan idiosincrásica, no sólo ha contribuido a la pérdida de peso y valor que padece en el ámbito de las ciencias sociales, sino también a la pérdida de identidad disciplinaria. Pues, fuera de los estrechos límites de nuestra profesión, ¿qué es un sociólogo para nuestros conciudadanos? ¿Es un

\footnotetext{
${ }^{4}$ Basta una breve visita a los stands de sociología de las principales librerías para darse cuenta de que están prácticamente monopolizados por discursos metafóricos como los de la "sociedad líquida" de ensayistas como Bauman, por ininteligibles juegos de palabras como los de Luhmann, o por otras lindezas similares de las que lo mínimo que se puede decir es que contribuyen más bien poco al avance del conocimiento científico-social (sin llevar a cabo tampoco una buena divulgación del existente).
} 
humanista, un pseudo-filósofo, un periodista con pretensiones, un activista político, un psicólogo aficionado, un ensayista ingenioso? ¿O es acaso, simplemente, un científico social? ${ }^{5}$

Mucho nos tememos que una parte de lo que se tiene por "sociología" ha abdicado de facto de la racionalidad y la ética de la ciencia en beneficio de un laxo y cómodo relativismo epistémico, incapaz (pues ni siquiera cree en la posibilidad) de generar conocimiento fiable, objetivo. Otra parte importante de nuestra disciplina, sin embargo, resiste estas tentaciones: sin duda existe muy buena sociología en gran parte del trabajo cotidiano de muchos investigadores sociales. El problema al que apuntamos es que, a diferencia de lo que ocurre en otras disciplinas, ese buen trabajo de muchos sociólogos coexiste con otros géneros de discurso y otras prácticas que se pretenden "sociológicas" cuando a duras penas superarían un mínimo escrutinio crítico desde el punto de vista no ya del rigor científico, sino incluso de la comprensión lingüística y la precisión lógico-formal. Cuando se renuncia (como ideal regulativo) a la posibilidad de un saber objetivo, guiado por criterios de verdad, cualquier cosa vale, también los lenguajes privados. La ciencia, en cambio, es necesariamente pública, una actividad de puertas abiertas, inspeccionable y controlable en el espacio público de la Gesellschaft científica. La situación inversa, es decir, la de la fragmentación de esa sociedad abierta en multitud de pequeñas comunidades cerradas y autorreferenciales, tiene importantes y variados costes. Quizá el más importante de todos sea el hecho de que se rompa la coherencia interna de la disciplina, lo cual a su vez se refleja de varias maneras y en distintos escenarios.

Basten algunos ejemplos: los fondos de investigación, plazas, publicaciones y recursos científicos se distribuyen a veces de forma que "no se deje fuera ninguna sensibilidad", como si de una negociación política se tratase; los congresos de la disciplina reúnen una multiplicidad de discursos tan disímiles que la comunicación y la crítica resultan difíciles e infructuosas; la (supuestamente forzosa) "elección" de "paradigma" se convierte para cada sociólogo o estudiante en una cuestión de gusto, de ideología o de identidad grupal (como ser de un equipo de fútbol), en vez de suscitar un examen crítico y racional sobre la fertilidad de cada enfoque; el mito del carácter "multiparadigmático" de la sociología se convierte en lugar común, y con frecuencia se utiliza la elección de un supuesto "paradigma" como blindaje contra cualquier examen crítico (ya sea lógico-teórico o empírico); se es víctima incluso de típicas preferencias adaptativas cuando se asume (con orgullo a veces) que la sociología ni quiere ni puede ser una disciplina científica como las demás. Por si ello fuera poco, se suele acusar de

\footnotetext{
${ }^{5}$ Esta situación genera desde hace tiempo un recurrente debate sobre la naturaleza de la sociología, y un malestar en los científicos sociales que ven cómo la labor propiamente científica se mezcla y confunde habitualmente con otros géneros de discurso: es el caso de lo que Boudon (2004) llama "sociología expresiva", o de lo que Goldthorpe (2004:123) denomina escritos de "revelación social", de "poesía social", o pretendsocial science; o, en fin, de lo que Elster (2007) ha bautizado como soft social science. Véase también Abell y Reyniers (2000).
} 
dogmático, "imperialista" o "positivista" cualquier intento de construcción de un suelo común basado en valores, hábitos, ideas y creencias compartidas, y en un lenguaje depurado de veleidades puramente retóricas.

Con la pérdida de cientificidad resultante de todo ello, la sociología pierde lo que seguramente sea su principal razón de ser: el potencial emancipador del conocimiento social. Volveremos sobre ello.

\section{Por Un GIRO ANALÍtICO EN SOCIOLOGÍA}

En este contexto se hace necesario un giro analíitico en sociología. La sociología analítica no es una teoría única ni un conjunto cerrado de teorías, sino un enfoque general para las ciencias sociales lo suficientemente abierto como para dar cabida a teorías de diferente alcance y para aceptar un razonable pluralismo metodológico, pero reconocible por la precisión conceptual, el rigor lógico, la apuesta por la construcción teórica y la contrastación empírica de las teorías. En palabras de uno de sus principales defensores, Peter Hedström, la sociología analítica busca explicar causalmente procesos sociales complejos diseccionándolos cuidadosamente para estudiar sus componentes fundamentales (de ahí el adjetivo "analítica"). ${ }^{6}$

Quizá lo que mejor define a esta sociología sea un "estilo" o "talante" analítico, un "aire de familia" (por decirlo con Wittgenstein) que todos los científicos sociales analíticos comparten; este talante incluye, entre otros rasgos, los siguientes:

- La precisión en las definiciones y la reducción de la ambigüedad, evitando los juegos de palabras supuestamente ingeniosos que no aportan información alguna, y estableciendo con nitidez el significado de los términos y conceptos utilizados. Sólo cuando todos usamos conceptos clara y distintamente definidos puede facilitarse la replicabilidad de resultados o su refutación, y puede florecer la discusión científica sustantiva, en vez de la mera discusión por palabras.

- La preocupación por la racionalidad del discurso y por el rigor lógico de la argumentación. El "talante analítico" no busca primordialmente "sugerir", sino explicitar todos y cada uno de los pasos lógicos de una cadena argumental, así como asegurar la correcta trabazón entre ellos.

\footnotetext{
${ }^{6}$ Véase, por ejemplo, Hedström (2005) o Barbera (2004). La reducción de un proceso o fenómeno complejo en términos de sus componentes fundamentales (su "análisis") es una condición necesaria, pero no suficiente, de la operación inversa (o "síntesis"): la predicción de esos fenómenos complejos a partir del conocimiento de los elementos que los componen, e incluso su posible generación intencional manipulando esos elementos (Wilson, 1998: cap.5); sin embargo, en ciencias sociales, la enorme complejidad de los fenómenos macrosociales hace improbable que algún día resulte sistemáticamente posible esta segunda operación, pero no así la primera (como las técnicas de simulación multi-agente están mostrando en la actualidad; Hedström, 2005; González, 2006).
} 
- El análisis del lenguaje es, en este sentido, una herramienta ineludible de toda ciencia; pero aún lo es más en el caso de la sociología, pues sólo mediante el mismo podemos acceder al contenido proposicional de las creencias, deseos e intenciones de las personas. La sociología analítica puede y debe recurrir al instrumental de la lingüística o de la filosofía del lenguaje para comprender cabalmente la acción social, y ello al tiempo que combate la costumbre (hoy de moda) de reducir la realidad a "discurso", y evita, asimismo, los peores excesos de la filosofía analítica del siglo XX en términos de irrelevancia y puntillosidad.

- La actitud epistémica racionalista, en la mejor tradición sociológica, única actitud capaz de evitar el solipsismo o el esoterismo del discurso y de convertirlo en tarea colectiva de una comunidad científica que aspira al conocimiento seguro y acumulativo.

- La opción metodológica por la explicación causal, siempre que sea posible, en términos de mecanismos y microfundamentos, y el intento de erradicación de las numerosas "cajas negras" existentes en los discursos habituales de la ciencia social. Como se dijo, el adjetivo "analítica" hace referencia a la descomposición de procesos complejos en elementos más simples, con vistas a entender los mecanismos causales por los que éstos se imbrican y relacionan para componer procesos y fenómenos de enorme complejidad. La perspectiva de los "mecanismos sociales" que la mayoría de los sociólogos analíticos utilizan (y sobre la que se dirá algo más abajo) pretende facilitar esta tarea explicativa ineludible en toda disciplina científica. ${ }^{7}$

- La construcción teórica como tarea inseparable de la explicación científico-social. En realidad, la teoría está presente en muchos niveles distintos de la explicación. Por ejemplo, cualquier explicación intencional de una acción (como las que realiza la teoría de la elección racional, pero también muchas corrientes hermenéuticas, fenomenológicas o interaccionistas) atribuye a los sujetos intencionales preferencias, cogniciones e interpretaciones de la situación, y esa es una atribución que se hace desde alguna posición teórica (sea el supuesto de la maximización de utilidad, que se deriva de una teoría de la utilidad; o el de la satisfacción, que ha defendido Herbert Simon; o el de los mecanismos de interacción entre creencias y deseos que se dan en los casos de autoengaño y wishful thinking, que utilizan Elster o la teoría $\mathrm{DBO}^{8}$ de Hedström; o el de la consistencia cognitiva en la teoría de la racionalidad cognitiva de Boudon; o el de los sesgos y heurísticas evolutivamente formados que hoy estudian la psicología y la economía experimentales, que deriva de una teoría evolutiva de la mente humana). El mismo tratamiento estadístico de datos y la misma contrastación empírica de teorías sería impensable sin la teoría subyacente de la probabilidad. La ciencia es teoría. Por ello, la sociología analítica también está a favor de los dos puntos siguientes:

\footnotetext{
${ }^{7}$ Sobre mecanismos sociales, véase Hedström y Swedberg (1998), Boudon (1998), Elster (2007, 1991) o Barbera (2004).

${ }^{8} \mathrm{DBO}$ son las iniciales en inglés de deseos (desires), creencias (beliefs) y oportunidades (opportunities); véase Hedström $(2005,2006)$.
} 
- La formalización y el uso de modelos formales como herramientas útiles para la teorización y la explicación científica, que aportan ventajas tales como disciplina, sistematicidad, exhaustividad, economía intelectual, ahorro de tiempo y esfuerzo, replicabilidad de resultados y explicitación clara de supuestos. Obviamente, e interesa subrayarlo, la modelización y la formalización no son más que un medio, nunca un fin de la investigación. El objetivo de la sociología analítica es siempre la producción de conocimiento, no la ostentación de habilidades técnicas; por esa razón, la sociología analítica no resuelve el trade-off entre simplicidad de los modelos y realismo explicativo inclinándose exclusivamente hacia la primera (como es el caso de los modelos de la economía neoclásica). ${ }^{9}$ La formalización (y en muchos casos, la matematización) como fin en sí mismo es otra vía abierta al oscurantismo y a la insustancialidad.

- La necesidad de tomar en serio los progresos y descubrimientos de las ciencias contemporáneas, de atreverse a considerar su relevancia para las ciencias sociales, y de buscar la integración de éstas dentro de la gran república de las ciencias, sin pretender permanecer aislados e impasibles en una suerte de islote académico. Hoy no podemos permitirnos hacer sociología de espaldas al resto de la ciencia ni a los logros contemporáneos de la teoría de juegos evolutiva, la teoría de la acción racional enriquecida conductualmente, la sociología y la economía experimentales, la psicología y la biología evolutivas, las ciencias cognitivas, el estudio neuro-psico-sociológico de las emociones, la teoría de la emergencia y evolución de las normas sociales, la teoría de las redes sociales, la ontología social analítica, etc. Muchas de las aportaciones teóricas y empíricas procedentes de otras tradiciones (incluso de obras no científicas) pueden tener, sin duda, una interpretación o un uso científicamente aceptable y sugerir buenas ideas a la investigación social; pero para ello es necesario formularlas de un modo claro, dentro de modelos teóricos donde los términos estén definidos con precisión, las relaciones causales bien establecidas y en lo posible comprendidas mediante el análisis de sus microfundamentos.

Los anteriores principios, que, lamentablemente, llaman hoy a escándalo a no pocos sociólogos, resultan obvios y redundantes en disciplinas científicas más consolidadas, hasta el punto de que nadie necesita añadir el adjetivo "analítico" a su tarea: la ciencia es "analítica" por definición y cultiva todas esas virtudes como parte de su propia estructura interna. Que una parte nada desdeñable de la sociología haya abandonado ese molde en que se reconocen las demás ciencias, también las sociales, es lo que recomienda añadir un adjetivo ("analítica") que de otro modo resultaría redundante aplicado a una disciplina científica. Y conviene aquí hacer dos observaciones: la primera, que no pretendemos estar diciendo nada excesivamente original cuando defendemos la sociología analítica como buena práctica científica: es sólo la notable extensión en nuestra profesión de

\footnotetext{
${ }^{9}$ Pueden verse dos potentes argumentaciones a favor del realismo explicativo y contra la simplificación excesiva de algunos modelos formales en Elster (2007:24-26 y 461-464) y Hedström (2005: 60-66 y 107-108).
} 
prácticas y discursos que niegan implícita o explícitamente estos principios lo que hace necesario reafirmarlos; la segunda, que lo de menos es que a este "código de buenas prácticas" lo llamemos "analítico" o no (una vez más, tiene poco sentido discutir por palabras, en vez de por cuestiones sustantivas): más allá del porqué del adjetivo (ya explicado más arriba), y del hecho de que sea el internacionalmente más aceptado para referirse a este talante, no tenemos ningún compromiso esencialista con el mismo.

\section{Contra ALgunAs CONFusiones fRECUENTES ACERCA DE LA SOCIOLOGÍA ANALítiCA}

La historia de los intentos más o menos oscurantistas de negar la posibilidad de una ciencia social (o natural) es muy antigua, y no vamos a detenernos aquí en este tipo de argumentos. Sin embargo, es también cierto que muchos sociólogos seria y honestamente comprometidos con el ideal de una ciencia social rigurosa y pública mantienen algunas reservas y objeciones frente al proyecto y los principios de la sociología analítica tal y como los hemos formulado. Creemos que algunas (si no la totalidad) de esas reservas pueden disiparse si se hacen algunas aclaraciones. Argumentaremos a continuación que, contrariamente a lo que a veces se escucha, la sociología analítica 1) es una perspectiva netamente antipositivista; 2) es teórica y metodológicamente pluralista (es más, defiende el auténtico pluralismo científico frente al pseudo-pluralismo); 3) es sensible al carácter histórico de la realidad social; 4) puede y debe ser sensible a las implicaciones y dimensiones éticas de la ciencia social.

\section{La SA es antipositivista}

La etiqueta de "positivista" se utiliza con mucha frecuencia en sociología como descalificación rápida de un punto de vista, a menudo sin explicitar claramente una definición precisa de la misma. No obstante, salta a la vista para cualquier persona mínimamente informada que numerosos rasgos y principios de la sociología analítica la sitúan en los antípodas de tales posiciones, al menos si nos atenemos a una definición de "positivismo" que tenga un mínimo sentido y que se corresponda con lo que históricamente ha recibido ese nombre en ciencias sociales. ${ }^{10}$

Sin ánimo de ser exhaustivos, aclaremos al menos los siguientes puntos: a) La sociología analítica atribuye estados mentales (como creencias, deseos o intenciones) a los agentes con objeto de explicar su conducta: es, en este sentido, sociología "comprensiva"enel más purosentido antipositivistaweberiano(aunqueesa"comprensión",

\footnotetext{
${ }^{10}$ Por ejemplo, el positivismo de Comte, el positivismo lógico del Círculo de Viena y sus seguidores, el conductismo, la investigación social empírica que busca correlaciones estadísticas ateóricamente, o incluso cualquiera de los sentidos en los que la Escuela de Frankfurt utilizaba el término (Adorno, 1972).
} 
como defendía el propio Weber, se conciba como un paso de la explicación causal, y no como un fin en sí mismo, como parecen pretender algunas escuelas autodenominadas "comprensivas"). b) Lo anterior tiene un corolario: la sociología analítica admite que entidades o hechos no directamente observables (y, por tanto, que no son directamente reducibles a "datos de los sentidos") formen parte esencial de una explicación científicosocial: los estados mentales son hechos de ese tipo; un conductista, o un positivista lógico, jamás admitirían ese papel para los mismos. c) En el núcleo de la sociología analítica está el rechazo de todo empirismo chato (practicado por ciertos positivismos) que intente basar las explicaciones sociológicas en meras correlaciones estadísticas entre variables, por mucha sofisticación técnica que se les ponga; la sociología analítica busca la especificación teórica de mecanismos causales y la construcción de modelos teóricos, sin contentarse con el puro descriptivismo estadístico. d) Precisamente la búsqueda de mecanismos sociales está muy alejada de la postulación de "leyes sociales" que los distintos positivismos siempre han defendido; los mecanismos sociales, como ha mostrado la sociología analítica, no son regularidades nomológicas, pero no por ello dejan de ser buenas explicaciones causales de multitud de fenómenos: en la ciencia social y en la psicología trabajamos con una concepción local de la causalidad (Davidson, 1964), que un positivista nunca consideraría científicamente aceptable. No obstante, cuando los modelos teóricos, con sus mecanismos explicativos localmente dirigidos, encuentran numerosos modelos empíricos que los satisfacen, entonces tenemos indicios para pensar que hay una regularidad social cuya lógica ha sido desentrañada. f) Finalmente, y por si lo anterior fuese poco, las connotaciones ideológico-políticas que a veces se han atribuido al positivismo están fuera de lugar en el caso de la sociología analítica, como veremos más abajo.

En resumidas cuentas, sorprende que a veces se use la etiqueta, con ánimo recusatorio, contra la sociología analítica, si no es desde el deseo de desprestigiarla asociándole un término (el de "positivista") que en rigor no se le puede aplicar, pero que, eso sí, es muy impopular en la comunidad sociológica.

\section{La SA es teórica y metodológicamente pluralista}

En primer lugar, el pluralismo teórico, la existencia de diversas teorías que compiten por explicar los mismos hechos o tipos de fenómenos, es algo consustancial a toda ciencia: la competición entre distintas teorías, en términos empíricamente decidibles, es una condición de posibilidad del progreso científico. Por ello una de las tareas fundamentales de los científicos es formular explicaciones alternativas de los fenómenos bajo estudio, que permitan explorar nuevas posibilidades y examinar críticamente la evidencia disponible. Este saludable y necesario pluralismo teórico, sin embargo, no tiene nada que ver con lo que usualmente se considera como tal en sociología, y que es, más bien, pseudo-pluralismo: la "coexistencia pacífica" entre diversas teorías autorreferentes, inasequibles a la evidencia empírica y a la crítica desde otras posiciones teóricas, y 
cultivadas por comunidades cerradas de adeptos, cual si de creencias religiosas o clubs de fans se tratase. ${ }^{11}$

El auténtico pluralismo teórico, el que hace competir las teorías entre sí convirtiendo las disputas en algo decidible racionalmente, ha hecho avanzar en los últimos años campos de investigación tan importantes para la sociología como el estudio del capital social, de la acción colectiva o de la movilidad social, por citar sólo algunos. En cambio, el pseudo-pluralismo teórico tiene su tragicómico reverso en la futilidad de los omnipresentes (y frecuentemente abstrusos) intentos de "superación de dicotomías teóricas", que a la postre acaban dejándolo todo igual, excepto por la creación de una corte de comentaristas, exégetas y críticos amigables. ${ }^{12}$ Los cultivadores de tales pseudo-discusiones se resisten a determinar qué cuenta como evidencia a favor o en contra de una teoría, y a entender las teorías no como compromisos vitales, identitarios o ideológicos (como cosmovisiones, al fin y al cabo), sino como modelos explicativos alternativos de un mismo fenómeno o rango de fenómenos, revisables y refutables a la luz de la evidencia empírica potencial.

Junto al pluralismo teórico, la sociología analítica defiende un sano pluralismo metodológico. El pluralismo metodológico, si es sensato y autoconsciente, no sólo es defendible sino inevitable en ciencias sociales. Al fin y al cabo, el mundo social está constituido por sujetos intencionales sometidos a un sinfín de determinaciones causales. Y en ese vértice causal e intencional, los sujetos producen sentido, se interpretan a sí mismos y al mundo circundante. Esto permite toda una serie de aproximaciones metodológicas científicamente útiles (todas ellas utilizadas en sociología analítica): desde el intencionalismo al estudio de causas no intencionales, desde la reconstrucción histórica al método comparativo, desde el diseño cuantitativo al cualitativo, ${ }^{13}$ desde el estudio de casos o la etnografía rigurosa (existe una "etnografía analítica"14) a la investigación demoscópica teóricamente orientada, desde la simulación multi-agente a la experimentación conductual. Al mismo tiempo, se puede defender analíticamente una postura individualista o bien ciertos tipos de holismo. A nadie se le escapa el enorme

${ }^{11}$ Goldthorpe (2000:7), por ejemplo, observa que el auténtico pluralismo "debe ir acompañado de una vigorosa competición entre ideas, que a menudo sea incluso mortal. Por el contrario, el pseudo-pluralismo se caracteriza por el hecho de que 'la confrontación de puntos de vista' no se organiza como una competición, ya sea porque las escuelas rivales saben protegerse contra los ataques 'ajenos', ya porque sus 'aproximaciones' se formulan de tal forma que resulten inmunes en gran medida a todo tipo de crítica". Para evitar esta situación debe existir un "consenso metodológico básico que determine cuáles son las reglas fundamentales y los criterios de éxito de acuerdo con los cuales debe guiarse la competición entre ideas".

12 Este argumento puede encontrarse desarrollado en Van den Berg (1998), Barbera (2004, 2006) 0 Goldthorpe (2000, 2007).

${ }^{13}$ Véase King, Keohane y Verba (1994); Abell (1988); Lago (2008); el propio Elster afirma que "lo que se puede denominar como 'el giro analítico' en ciencias sociales no descansa, a mi modo de ver, en el uso de metodología cuantitativa, sino en un interés casi obsesivo por la claridad y la explicitud" (2007: 455).

${ }^{14}$ Véase una panorámica a este respecto en Vaughan (2009). 
potencial del llamado action pattern (paradigma de la acción) y de las explicaciones intencionales en ciencia social. Con todo, algunos teóricos sociales analíticos aceptan que puede haber espacio para algún tipo de explicación funcional de naturaleza evolutiva, que postule la existencia de mecanismos de equilibración por consecuencias activados por atractores evolutivos ya sean de selección natural (como en el submodelo sociobiológico), ya de refuerzo (como en el submodelo lingüístico). Obsérvese, no obstante, que dichas explicaciones evolutivas no operan con causas finales sino con causas eficientes (como por otra parte, lo hace la teoría de la evolución en biología), y que, para ser legítimas, deben estar nítidamente especificados los mecanismos causales estándar que permiten la persistencia de un determinado rasgo o fenómeno (que lo "seleccionan") por sus consecuencias en un contexto dado. ${ }^{15}$

En cualquier caso, es posible asimismo sostener -como Goldthorpe (2000) o King, Keohane y Verba (1994) - que todas estas opciones metodológicas siguen una misma lógica de inferencia científica. De lo que desde luego no es partidaria la sociología analítica es de la arbitrariedad y del "todo vale" que aún impera en algunos círculos académicos.

\section{La SA es sensible a la naturaleza histórica de la realidad social}

Las ciencias sociales tienen una insoslayable dimensión histórica ${ }^{16}$ No sólo porque los individuos (los sujetos intencionales) tenemos memoria, sino porque el conjunto de normas e instituciones que engendramos en la interacción social también tiene "memoria" y pasado. De hecho, una clave fundamental para entender cualquier institución social (el mercado, la democracia, el dinero, el sistema bancario o la escuela) es entender la historia acumulativa de sus adaptaciones locales. En esa historia evolutiva el científico social descubrirá un sinfín de consecuencias no intencionadas de miríadas de acciones intencionales, y observará cómo la evolución adaptativa y las intenciones trabajan conjuntamente en la génesis y estructuración de las instituciones sociales, instituciones que nadie diseñó ex novo y cuyas futuras transformaciones son difíciles de prever. Pero descubrirá también que las instituciones no evolucionan aisladamente, sino que co-evolucionan formando complejos sistemas en equilibrio siempre relativamente inestable y, por lo tanto, siempre dinámico.

${ }^{15}$ Sobre la legitimidad de las explicaciones evolutivas en ciencias sociales véanse Van Parijs (1984) y Faia (1986). Véase asimismo el debate sobre individualismo metodológico y explicación funcional mantenido por esta cuestión entre los marxistas analíticos, en el que participaron Cohen (1984), Van Parijs (1984) y Elster (1984); o las posturas anti-individualistas de Pettit (1993) o Levine, Sober y Wright (1987), entre otros teóricos analíticos. De hecho, uno de los campos más interesantes de la filosofía de la ciencia social es hoy el de la ontología social, inexplicablemente descuidado en la tradición sociológica.

${ }^{16}$ En realidad, como sabía Marx, todas las ciencias la tienen, aunque la escala temporal a la que cambian los fenómenos bajo estudio varía sobremanera entre ellas, de manera que podemos hacer la ficción de considerar que algunas son más "históricas" que otras. 
La sociología histórica es un complejo programa de investigación desarrollado por la sociología, desde sus mismos inicios, precisamente para abordar esos procesos de coevolución institucional. Pues bien, desde la perspectiva analítica aquí defendida entendemos que la sociología histórica, comparativa o no, que estudia todos estos procesos a nivel micro y macrosocial, puede ser tan científica como cualquier otra subdisciplina. ${ }^{17}$ En general, los mismos principios que la sociología analítica propone para el estudio de los fenómenos sociales presentes pueden ser aplicados sin más para el de cualesquiera fenómenos históricos. Es verdad que la sociología histórica tiene limitaciones metodológicas intrínsecas que el análisis comparado no puede erradicar, como ya señaló Max Weber en su día, y como Goldthorpe volvió a recalcar en el célebre debate de los noventa. ${ }^{18}$ Estas limitaciones tienen que ver con la naturaleza de segundo grado del saber socio-histórico y con la "libertad" del sociólogo histórico para elegir las interpretaciones historiográficas que mejor se amolden a su esquema general. Es cierto que existe esa libertad, pero hay modos de intentar controlarla científicamente, y el propio pluralismo teórico impone un control y una limitación de la misma. Como en general ocurre en la ciencia, mientras no tengamos una teoría mejor o no se demuestre suficientemente qué hechos fundamentales quedan inexplicados o marginados, la teoría vigente debe prevalecer. Por ejemplo, la teoría de Rueschmeyer, Stephens y Stephens (1992) corrige la de Barrington Moore ("sin burguesía no hay democracia") a la hora de explicar las trayectorias democráticas europeas, con una tesis alternativa: "sin proletariado no hay democracia". La teoría de Brenner (1988) sobre la transición al capitalismo corrige las teorías exógenas y deterministas proponiendo una explicación endógena y contingente basada en la lucha de clases. Habría otros muchos ejemplos.

Por lo demás, desde la propia sociología analítica están surgiendo avances que enriquecen la misma tradición de la sociología histórica. Citemos, a modo de buena ilustración, el reciente e interesantísimo debate sobre las analytical narratives (que intentan utilizar la elección racional enriquecida conductualmente en el estudio de la historia social y política), y los avances metodológicos a que ha dado lugar. ${ }^{19}$ Asimismo pueden citarse las aportaciones recientes en el campo del neoinstitucionalismo, las teorías de la path dependence, o la aplicación de la teoría de juegos evolutiva o las teorías de la evolución cultural al estudio del surgimiento histórico de normas e instituciones sociales, motivaciones pro-sociales o rasgos culturales.

Todo ello tiene cabida dentro de una buena sociología analítica que no sólo no ignora la historia sino que la considera un inagotable banco de datos y de fenómenos

\footnotetext{
${ }^{17}$ Así lo certifican, por ejemplo, los trabajos recientes de Tilly $(2006,2008)$ o el más clásico de Rueschmeyer, Stephens y Stephens (1992).

${ }^{18}$ Max Weber (1983: 11-22) y J. Goldthorpe (1991).

${ }^{19}$ Por ejemplo, Abell $(2007,2004,2000)$, Bates et. al. (2000, 1998), Gould (2007), Levi (2006), Schiemann (2007). La American Political Science Review (vol. 94, $n^{\circ} 3$ ) y la revista italiana Sociologica ( $n^{\circ} 3 / 2007$ ) han publicado sendos simposios monográficos sobre el tema.
} 
que explicar; incluso la historia puramente descriptiva nos proporciona datos e interpretaciones que una buena ciencia social puede insertar en modelos explicativos. Lo que sí nos parece rechazable, en todo caso, es la utilización de eslóganes sobre "la historicidad de lo social" como coartada para la ausencia de rigor metodológico y teórico y para el ensayismo apresurado y las interpretaciones sin base.

\section{La SA es sensible a las implicaciones éticas de la ciencia social}

Ahora bien, a la sociología analítica no le mueve únicamente una preocupación cognitiva o científica (aunque sea esa su principal función), sino también una inquietud ética 0 social. En primer lugar, por una cuestión de ética intelectual. La sociología debe dirigirse a la sociedad como una disciplina orgullosa de sí misma y segura de lo que dice, basándose en sólidos fundamentos teóricos y evidencias empíricas adecuadas. Los estudiantes no deben perder el tiempo y el esfuerzo descifrando intrincados y vacíos discursos, por muy atractivos y supuestamente "críticos" que pretendan ser. La auténtica crítica rechaza un tipo de discurso del que nunca se sabe con certeza lo que dice, ni con qué apoyos empíricos cuenta. El auténtico talante crítico suscribe las palabras de Dagfinn Føllesdal:

"Cuando tratamos de convencer a nuestros congéneres de que adopten nuestros puntos de vista, no deberíamos hacerlo ni mediante la coerción ni mediante el uso de recursos puramente retóricos: deberíamos inducirles a aceptar o rechazar nuestro punto de vista sobre la base de sus propias reflexiones. Esto sólo puede conseguirse mediante la argumentación racional, gracias a la cual se reconoce a la otra persona como una criatura autónoma y racional" (Føllesdal, 1996: 207)

En este sentido, el compromiso con la sociología analítica es también una cuestión de ética personal y social. El discurso inteligible y lógicamente ordenado, la argumentación y la justificación racional, la prueba empírica y el contraste con los hechos, se oponen a los discursos supuestamente seductores pero indiferentes a cualquier principio elemental de disciplina intelectual; estos últimos suelen desembocar en la esterilidad cognitiva, el tribalismo intelectual y el fetichismo del lenguaje. La buena sociología no es literatura (por buena que ésta pueda ser), ni periodismo sofisticado, ni tertulia de café, ni simple erudición o hagiografía de autores, ni investigación caprichosa de excentricidades irrelevantes, ni diario de impresiones o de viajes, ni traducción de trivialidades a lenguajes ampulosos, ni creación de etiquetas conceptuales para renombrar lo ya conocido.

Junto a la preocupación por la ética y la honestidad intelectual, la sociología analítica es plenamente compatible con cualquier inquietud por la ética y la justicia social. Muchos de entre quienes la cultivan lo hacen con la mirada puesta en la posible utilidad social de su tarea científica, y escogen sus temas y objetos de investigación teniendo en mente su relevancia para la realización de ciertos ideales distributivos igualitaristas (no cabe olvidar, por ejemplo, que ha existido y existe un "marxismo analítico", y que los científicos sociales que utilizan el enfoque que defendemos mantienen posiciones 
políticas a lo largo de todo el espectro ideológico). Conviene aclarar de inmediato que esta compatibilidad no implica ningún compromiso "esencial" de la sociología analítica en términos políticos; la utilidad social y política de la sociología, y el compromiso personal de quienes la cultiven, es perfectamente compatible con su "neutralidad valorativa" en el sentido weberiano, como no puede ser de otro modo en ciencia, si se quiere evitar el sesgo ideológico en el planteamiento de modelos teóricos, hipótesis, selección de casos y recogida e interpretación de datos.

¿Quiere esto decir que la sociología analítica como tal es insensible a las implicaciones éticas y políticas de la ciencia social? En absoluto. La sociología, como toda ciencia social, debe aspirar a explicar positivamente hechos y procesos sociales y generar conocimiento objetivo. Esto es cierto, sí, pero no es todo, porque la ciencia social debe tener una dimensión práctica, orientada también a la intervención social. ${ }^{20}$ Una buena sociología positiva, como la que reivindicamos aquí, hará buen diagnóstico social, puesto que construirá teorías y ofrecerá explicaciones causales de determinados hechos y fenómenos sociales que son necesarias e imprescindibles para cualquier intervención social sensata y para cualquier pronóstico social: como sabía el Marx que se encerró durante años en la biblioteca del Museo Británico, la acción práctica sostenida en el tiempo, si no está basada en un buen conocimiento teórico, está condenada al fracaso, por muy bienintencionada políticamente que sea.

Pero, sostenemos, la perspectiva analítica aún puede ir más allá en este terreno (más allá, por cierto, de lo que ninguna "sociología crítica" ha ido nunca): para intervenir en la sociedad, para contribuir al progreso social como saber práctico no bastan los diagnósticos y las explicaciones causales, hacen falta ideales o principios normativos y claridad sobre las razones que los sustentan. Nuestra tesis en este punto es que el rigor analítico que se le exige al conocimiento de lo que es, se le puede exigir también al conocimiento de lo que debe ser. Ambos tipos de conocimientos son fundamentales, perfectamente legítimos y pueden ser rigurosamente racionales. Así pues, el giro analítico de la sociología que aquí defendemos es también una invitación a desarrollar una sociología normativa con personalidad propia.

Si esto suena atrevido, baste recordar que la ciencia social más madura y refinada, la economía, posee una rama de saber económico fundamental para la disciplina, que es la llamada economía del bienestar. Los economistas no tienen inconveniente en llamar a esa parte de la disciplina economía normativa. Por resumir mucho, es un saber (muy técnico, por cierto) que gravita sobre un concepto normativo, un valor: la eficiencia, que Vilfredo Pareto consiguió formalizar y que se articula a partir de los conceptos aledaños de racionalidad y bienestar. Se podrían llenar páginas y páginas para contar hasta dónde

${ }^{20}$ Lo que no implica que no pueda y deba existir conocimiento científico-social inútil, cuyas posibles aplicaciones prácticas se desconocen. En toda ciencia existen conocimientos de este tipo: la teoría de la relatividad de Einstein, uno de los estandartes de la ciencia contemporánea, no ha conocido aún ni una sola aplicación práctica o tecnológica. 
han llegado los economistas con ese saber práctico por el camino de la intervención social. Por otro lado, también la filosofía analítica contemporánea ha desarrollado un saber práctico, a saber, la ética social normativa, que ha dado de sí uno de los debates más importantes de los últimos años sobre la buena sociedad y sobre la sociedad justa. En particular, la ética social de raíz rawlsiana ha sabido aislar una teoría (la justicia como equidad) que trabaja sobre los conceptos de libertad, igualdad y reciprocidad. Con ella, ciertamente, no ha influido tanto como los economistas; pero cuenta con esos conceptos, están bien definidos y articulados teóricamente y son políticamente muy poderosos. De hecho, ha penetrado en la propia economía normativa forzando a los propios economistas a revisar sus planteamientos y dar cabida a las demandas de la justicia: el caso de Amartya Sen es posiblemente el más relevante, pero en ningún caso el único.

¿Cuál es el concepto normativo de la sociología? En realidad, muchos y a la vez ninguno. Porque la sociología ha renunciado a desarrollarse como disciplina normativa en este sentido técnico riguroso. $Y$ de ahí que su capacidad real de influencia social sea menor de lo que debería y podría ser. Una tarea importante, por realizar, de la sociología analítica es precisamente desarrollar una rama normativa, articular un concepto (o familia de conceptos) con que la sociología podría organizar un saber normativo capaz de "discutir" en pie de igualdad con la ética social o la economía del bienestar. Esa familia de conceptos podría gravitar (es una mera sugerencia) en torno a una buena y precisa definición del concepto de cohesión social, y ordenar así el rico complejo conceptual elaborado por la tradición sociológica, un complejo que incluye conceptos como los de cooperación y confianza, solidaridad y reciprocidad, identidad y pertenencia, capital social y cumplimiento de normas, jerarquía y autoridad, etc., todos ellos susceptibles también de definiciones precisas, pues la cohesión social no es lo mismo que la justicia, y mucho menos que la eficiencia. Lograr una comunidad bien integrada, intervenir para crearla, consolidarla, presupone pero también va más allá de la redistribución de recursos materiales guiados por una ética social o una teoría de la justicia. Supone manejar recursos simbólicos, fomentar el sentido de pertenencia, elevar los niveles de capital social, combatir la atomización y la fragmentación social, lograr el cumplimiento de normas, evitar problemas de acción colectiva, estimular las disposiciones a la cooperación y disponer de diseños institucionales que cumplan ciertas condiciones y objetivos.

Nótese que una sociología analítica normativa como ésta estaría libre de herencias ideológicas específicas. El universo conceptual de la cohesión social puede tener muy diversas articulaciones teóricas y muy diversas consecuencias políticas. Lo que aquí proponemos (y no proponemos más que eso) es que ampliar el debate sobre la buena sociedad añadiéndole un eje sociológico-normativo supondría un claro enriquecimiento de dicho debate. Permitiría, en efecto, plantear interesantes problemas de trade-off entre los principios mencionados. Así, dependiendo de esos trade-offs obtendríamos diferentes modelos de buena sociedad con distintos equilibrios entre eficiencia, justicia y cohesión (o cualquier otro gran principio sociológico-normativo que eligiéramos desarrollar). 


\section{Conclusión}

En resumen, las más de las veces el desprecio apresurado del punto de vista analítico en sociología, basado en acusaciones como las de "positivismo", "intolerancia teórica y metodológica", "olvido de la historia" u "olvido de la crítica social", ha servido simplemente como excusa para mantenerse cómodamente instalados en una sociología acientífica. La sociología analítica busca precisamente plantear todas esas preocupaciones de modo compatible con la cientificidad y el avance del conocimiento racional, pues, como bien sabía John Harsanyi,

"No hay incompatibilidad de ningún tipo entre el pensamiento claro y preciso, en las mejores tradiciones de las ciencias matemáticas, y una profunda preocupación por los valores humanos en las mejores tradiciones de las humanidades y la filosofía política clásica" (Harsanyi, 1969: 537).

Para que la sociología recupere su prestigio frente a ciertas corrientes que la han vuelto trivial y prescindible es necesario que dé, pues, un giro analítico. Ya hemos dicho que el giro analítico no supone algo conceptual o científicamente novedoso como tal; es más, implica en gran medida una vuelta atrás, un retorno a los fundamentos clásicos de la disciplina. Pero, en el contexto institucional y académico de la sociología, presenta también aspectos novedosos: la apuesta por la interdisciplinariedad (estableciendo relaciones incluso con la biología, tradicionalmente estigmatizada por los sociólogos); la utilización de técnicas de investigación innovadoras (como la simulación multi-agente o la experimentación conductual); la refrescante perspectiva teórico-metodológica de los mecanismos sociales; la introducción de una perspectiva evolutiva en la ciencia social; el desarrollo de nuevas teorías y fructíferos campos de investigación acumulativa. Todo ello nos hace albergar esperanzas sobre un prometedor futuro para el "programa" analítico.

El giro analítico que defendemos no busca crear ortodoxia o dogma alguno. Bien al contrario: busca combatir los existentes y conseguir que la sociología sea un campo intelectual en la vanguardia de la lucha contra las ortodoxias, dogmas y oscurantismos varios que con frecuencia amenazan al intelecto humano; querer mantener los propios universos discursivos como reinos de taifas impertérritos a la crítica externa e impermeables a la evidencia empírica y el progreso del conocimiento: en eso consiste el auténtico dogmatismo (aunque a veces se vista de "crítico" o de ir "a contracorriente").

Para terminar conviene volver de nuevo a Weber. En Economía y sociedad define así la sociología: "una ciencia que pretende entender, interpretándola, la acción social para de esa manera explicarla causalmente en su desarrollo y efectos" (1922: 5). Este y no otro es el objetivo de la sociología analítica. Estos son los fundamentos a los que debe retornar la sociología. Bien anclada en esa base podrá dialogar entonces con la mejor ciencia de hoy; podrá desarrollar una rama normativa propia que contribuya al desarrollo de una sociedad no sólo más eficiente, sino mejor, esto es, más justa e integrada; podrá, en fin, sentirse satisfecha de la enorme cantidad de conocimiento teórico y empírico sobre la sociedad que ha sido capaz de acumular en poco más de siglo y medio. 
La ciencia (también la ciencia normativa) nos libera de la ignorancia y confiere al ser humano un enorme poder de transformación de la realidad; un poder (todo hay que decirlo) tan grande que a menudo nos ciega, y que puede también tener consecuencias negativas y ser susceptible de usos éticamente cuestionables: como sabía Manuel Sacristán, es el buen conocimiento el que resulta peligroso. ${ }^{21}$ Pero abdicar del saber o renunciar a la racionalidad científica no nos va a situar en mejor posición para hacer frente a los problemas de la humanidad, sino todo lo contrario: por decirlo con Mosterín (2000), sería tan absurdo como cortarnos las manos porque pueden servir tanto para acariciar como para golpear.

\section{BibLiografía}

Abell, P. (1988), The Syntax of Social Life. Oxford: Oxford University Press.

(2000), "Putting Social Theory Right?", Sociological Theory, 18: 518-523

(2004), "Narrative Explanation: An Alternative to Variable-Centered Explanation?", Annual Review of Sociology, 30: 287-310.

(2007), "Narratives, Bayesian Narratives and Narrative Actions", Sociologica, no 3/2007 (http://www. sociologica.mulino.it).

Abell, P. \& Reyniers, D. (2000), "On the failure of social theory", British Journal of Sociology, 54: 739-750.

Adorno, Th. W. (ed.) (1972), La disputa del positivismo en la sociología alemana. Barcelona: Grijalbo,.

Barbera, F. (2004), Meccanismi sociali. Elementi di sociologia analitica. Bolonia: II Mulino.

(2006), "A Star is Born? The Authors, Principles and Objectives of Analytical Sociology", Papers. Revista de Sociologia, 80: 31-50 (http://ddd.uab.cat/pub/papers/02102862n80p31.pdf).

Bates, R. H., A. Greif, M. Levi, J.L. Rosenthal y B.R. Weingast (2000), "The Analytical Narrative Project", American Political Science Review, 94: 696-702.

Bates, R. H.; Greif, A.; Levi, M.; Rosenthal, J.-L. y Weingast, B. R. (1998). Analytic Narratives. Princeton (NJ): Princeton University Press.

Boudon, R. (1998), "Social mechanisms without black boxes", en Hedström, P. y Swedberd, R. (eds.), Social mechanisms. An Analytical Approach to Social Theory. Nueva York: Cambridge University Press, 172203.

${ }^{21}$ Véase Sacristán (1983:201), "lo característico de la tecnociencia contemporánea (como de todo conocimiento, en realidad) no es una supuesta bondad o maldad, sino su constitutiva ambigüedad práctica"; todo producto científico "conlleva un riesgo probablemente proporcional a su calidad epistemológica". 
(2003), Raison, bonnes raisons. París: PUF.

(2004), "La sociología que realmente importa", Papers. Revista de Sociologia 72: 215-226.

Brenner, R. (1988), "Estructura de clases agraria y desarrollo económico en la Europa preindustrial", en T.H. Aston y C.H. E. Philpin, comps. (1988), El debate Brenner, Barcelona. Crítica, pp. 21-81.

Cohen, G. A. (1984). "Réplica a «Marxismo, funcionalismo y teoría de juegos» de Elster", Zona Abierta, 33: 63-80

Davidson, D. (1963). "Actions, Reasons and Causes", en Essays on Actions and Events. Oxford: Oxford University Press, 1980, pp.3-20.

Elster, J. (1984), "Marxismo, funcionalismo y teoría de juegos. Alegato en favor del individualismo metodológico", Zona Abierta 33: 21-62.

(1991), Tuercas y tornillos. Una introducción a los conceptos básicos de las ciencias sociales. Barcelona, Gedisa.

(2007), Explaining Social Behavior. Nueva York: Cambridge University Press.

Faia, M. (1986), Dynamic Functionalism: Strategy and Tactics. Cambridge: Cambridge University Press.

Føllesdal, D. (1996), "Analytic philosophy: what is it and why should one engage in it?", Ratio 9: 193-208.

Goldthorpe, J. H. (1991), "The uses of history in sociology: reflections on some recent tendencies", British Journal of Sociology 42: 211-230.

(2000), On Sociology. Numbers, Narratives and the Integration of Research and Theory. Oxford: Oxford University Press.

(2004), "The Scientific Study of Society", British Journal of Sociology 55: 123-126.

(2007), On Sociology (Second Edition). Vol. 1: Critique and Program. Stanford: Stanford University Press.

González, S. (2006), "The Role of Dynamic Networks in Social Capital: A Simulation Experiment", Papers. Revista de Sociología 69: 171-194.

Gould, R.V. (ed.) (2007). The Rational Choice Controversy in Historical Sociology, Chicago: Chicago University Press.

Harsanyi, J.C. (1969), "Rational-Choice Models of Political Behavior vs. Functionalist and Conformist Theories", World Politics 21: 513-538.

Hedström, P. (2005), Dissecting the Social. On the Principles of Analytical Sociology. Cambridge: Cambridge University Press.

(2006), "Explaining Social Change: An Analytical Approach", Papers. Revista de Sociologia, 80: 73-95 (http://ddd.uab.cat/pub/papers/ 02102862n80p73.pdf) 
Hedström, P. y Bearman, P. (eds., en prensa) (2009), The Oxford Handbook of Analytical Sociology. Oxford: Oxford University Press.

Hedström, P. \& Swedberg, R. (eds.) (1998), Social Mechanisms. An Analytical Approach to Social Theory. Cambridge: Cambridge University Press.

King, G., Keohane, R.O. y Verba, S. (1994), Designing social inquiry: scientific inference in qualitative research. Princeton (N.J.), Princeton University Press.

Lago, I. (2008), La lógica de la explicación en las ciencias sociales. Madrid: Alianza Editorial.

Levi, M. (2006), "Modelando procesos históricos complejos con narrativas analíticas", Revista Uruguaya de Ciencia Política 15: 11-29.

Levine, A., Sober, E. y Wright, O.E. (1987), "Marxismo e individualismo metodológico", Zona abierta, 41-42: 131-158.

Mosterín, J. (2000), "Ciencia, filosofía y humanidades", Pasajes. Revista de Pensamiento Contemporáneo, $n^{0} 4$ (septiembre).

Pettit, Ph. (1993), The Common Mind: An Essay on Psychology, Society and Politics, Nueva York: Oxford University Press.

Rueschmeyer, D., Stepehens, E.H. y Stephens J.D. (1992), Capitalist development and democracy, Cambridge: Polity Press.

Sacristán, M. (1983), “Entrevista con Manuel Sacristán”, Mientras tanto 16-17: 195-211.

Schiemann, J.W. (2007), "Bizarre Beliefs and Rational Choices: A Behavioral Approach to Analytic Narratives", Journal of Politics, 69: 511-524.

Tilly, Ch. (2006), Why? What Happens When People Give Reasons... and Why. Princeton: Princeton University Press.

(2008), Explaining Social Processes. Boulder (CO): Paradigm Press.

Van den Berg, A. (1998), "Is Sociological Theory Too Grand for Social Mechanisms?", in Hedström, Peter \& Swedberg, Richard (eds.): Social Mechanisms. An Analytical Approach to Social Theory. Cambridge: Cambridge University Press, pp. 204-237.

Van Parijs, Ph. (1982), Evolutionary Explanations in the Social Sciences, Totowa, New Jersey: Rowman and Littlefield.

(1984), "El marxismo funcionalista rehabilitado. Comentario sobre Elster", Zona Abierta, 33: 81-102.

Vaughan, D. (2009, en prensa), "Ethnographic Analytics", en P. Hedström y P. Bearman (eds.), The Oxford Handbook of Analytical Sociology. Oxford: Oxford University Press.

Weber, M. (1983 [1915-1919]), Ensayos sobre sociología de la religión (vol. 1). Madrid: Taurus. 
(1964 [1922]), Economía y sociedad. Esbozo de sociología comprensiva, México: Fondo de Cultura Económica.

RECIBIDO: $2 / 11 / 08$

ACEPTADO: 26/11/08

RIS, VOL. 67, No 2, MAYO-AGOSTO, 437-456, 2009. ISSN: 0034-9712 